\title{
Affordable Software for Collaboration, Document Management, and on-site Information Management in Small- and Medium-sized Construction Companies
}

\author{
Mehdi Nourbakhsh, Samaneh Zolfagharian, Rosli Mohamad Zin, and Javier Irizarry
}

\begin{abstract}
Small- and medium-sized enterprises (SMEs) which account for 99 percent of construction companies in Europe cannot afford to invest in complex technological solutions. Instead, they prefer to interact with a very simple mechanism that can help them to improve their business. It has been reported that the most frequently identified issue in construction industry is related to collaboration and disintegration of different participants. The aim of this study is to identify affordable current market's software for collaborative team working, document management, and on-site information management in small- and medium-sized construction companies (SMCCs). Such software should be an affordable tool for collaboration, on-site information management, and document management in SMEs. Based on the results of interviews with 12 IT professionals, the selected software was 'DropBox'. Simplicity, functionality, security, and affordability are the main features of this software. The result of this study is useful for SMCCs to implement information technology in their companies.
\end{abstract}

Index Terms-Collaboration, document management, information management, information sharing.

\section{INTRODUCTION}

Large companies have significant impact in the construction industry and are many times reluctant to employ labours directly. Instead they engage professionals and experts by hiring subcontractors to deliver the projects. Therefore, each project needs participation of several smalland medium-sized subcontractor companies. Temporary relationships among these parties cause spending time and resources in integration of process and information. This problem of disintegration in the construction industry which has been explored by researchers for two decades still remains unsolved [1].

On the other hand, construction personnel needs to access large amounts of information ranging from project design drawings to personal diaries to support their ongoing works and to make decisions about the process of construction [2]. It has been reported that the most frequently identified issue in the construction industry is related to collaboration (including communications, document management, and

Manuscript received May 15, 2012; revised June 18, 2012. This work was supported in part by the Universiti Teknologi Malaysia (UTM) under the project vote number 77318 .

Mehdi Nourbakhsh and Javier Irizarry are with School of Building Construction, Georgia Institute of Technology, Atlanta, Georgia, USA (e-mail: mehdi.nourbakhsh@gatech.edu)

Samaneh Zolfagharian and Rosli Mohamad Zin are with Faculty of Civil Engineering, Universiti Teknologi Malaysia (UTM), Skudai, Malaysia. interoperability) [3]. Because of the intensity and diversity of construction information and participation of various parties, collaborative teamwork is crucial to the construction industry.

Although advances in information technology (IT) provide the tools to address the above problems [4, 5], significant investments are required from construction companies. However, large companies often have enough IT budgets and they can invest and benefit from IT in their projects, small companies cannot afford similar investments in this area due to high expense of purchasing disparate systems that eventually need to be replaced [6]. In fact smalland medium-sized enterprises (SMEs) are not interested in the complexity of the technological solution but prefer to interact with very simple mechanisms that can help them to improve their business [7]. There are plenty of commercial products for this purpose, they are all technology-driven, very specific and lack simplicity and functionality [6]. Ease of use/user friendliness plays an important role in software selection of SMEs because they lack technical personnel to work in this area [8]. The aim of this study is to identify affordable current market's software for collaborative teamwork, document management, and on-site information management in small- and medium-sized construction companies.

\section{LITERATURE REVIEW}

Collaboration is not apart from communication and information management. Since collaboration is an important issue, information management would also be important. Early studies have classified construction information. Scott and Assadi [9] defined three categories, namely financial, progress and quality. De la Garza and Howitt [10] classified it into 10 groups which are requests for information, material management, equipment management, cost management, schedule means and methods, jobsite record keeping, submittals, safety, quality control (QC) /quality assurance (QA), and future trends. Tenah [11] had a different point of view and he defined the information needs of project participants based on their roles and responsibilities. It can be seen that different researchers had different perspectives on categorization of construction information and there is no unique classification of information. It conforms to nature of information in construction site while every construction site has its own information classification and it differs from one to another.

Significant research studies have explored the application 
of IT for managing construction information [12] including site diaries [13], resource management [14], supply chain management [15], resolution of site design problem [16], defect management [17], quality inspections [18-19], maintenance conditions [20], health and safety [5], progress records [21] and monitoring [22].

Studies on application of IT in SMEs have been on-going for the last two decades. Forcada et al. [6] devised a web database for document management. Perera and Imriyas [23] proposed a system for time and cost management. Zhiliang et al. [24] developed a system for construction personnel collaboration by XML. Cheung et al. [25] explored a web based construction performance monitoring system (PPMS) while Chan and Leung [7] proposed a prototype for web based data exchange using XML technology.

In summary, these systems mostly rely on expertise for design and implementation, thus SMEs should engage expert personnel that may not be affordable for them. However, none has reported the use of a flexible and simple tool that could be customized and broadly used in various construction projects.

\section{Methodology}

Although there are plenty of commercial software for collaboration, document management, and on-site information management, they are not functional, simple, and affordable for SMEs. This study has investigated current commercial software to find appropriate software to be able to address the problem of disintegration in SMEs. An interview with an IT expert panel group including 12 IT professional was conducted in Malaysia to collect the data for this study. The IT professionals had more than eight years of experience working in small- and medium-sized construction companies. Afterwards, 46 available programs were investigated in order to find the best available commercial software for collaboration, document management, and on-site information management.

\section{ANALYSIS AND RESUlTS}

The result of the interview with expert panel shows the following criteria for software selection:

- Inadequate technical personnel causes SMEs prefer user-friendly software. Thus, selected software must be simple enough to be handled efficiently and without training by everyone who has basic knowledge of computer and operation system.

- The software must support collaborative teamwork, document management, and also on-site information management.

- The software must be affordable to be used by SMEs. Since the term 'affordability' differs from one company to another, the software that can be used for free is preferred.

- The software must be highly secure because it deals with the entire project's information. The term 'security' means that the company data should not be shared with unauthorised people. It also means that to keep data in a secure place to be retrieved at a proper time. Even if the
Projects' computer is broken down the project data must be maintained and recovered.

- The software must support template documents because many companies have developed their own templates and they prefer to use them.

- There is slow Internet connection in many developing countries. Even in countries with high speed Internet access, there is sometimes slow Internet connection in construction sites. Selected software must support slow Internet connection in order to be used broadly on construction sites. Therefore, synchronization must be performed automatically whenever the Internet connection is stable.

Based on the interview, it was found that the best alternative to achieve these criteria is the use of online backup programs and that the software has the following features; information sharing, mobile access, desktop client and automatic synchronization.

In addition, the expert panel group identified the following features to be as "Must" have; Affordability (AF), File/Folder Sharing (FS), Mobile Access (MA), Desktop Client (DC), Online Storage (OS), File/Folder Synchronization (SY) while following features were identified as "Preferred" to have; Free Storage Plan in Gigabyte (FP), Annual Cost per Gigabyte (ACG), Multi Computers Account (MC), Unlimited Bandwidth (UB), Unlimited Users (UU). To provide a quantitative measurement, the expert panel came out with +5 score for all preferred features, except 'Cost per Year' which had -10 score because of its importance.

Table 1 shows the software which comply with 'Must' have criteria. Those programs which did not comply were eliminated from the list. In addition, Table 2 illustrates the comparison of mentioned programs that comply with 'Preferred' criteria.

\begin{tabular}{llllllll}
\multicolumn{7}{c}{ TABLE I: TOP RANKED SOFTWARE COMPLY WITH 'MUST' HAVE } \\
\hline \hline Rank & Service Provider & AF & FS & MA & DC & OS & SY \\
\hline 1 & DropBox & $\sqrt{ }$ & $\sqrt{ }$ & $\sqrt{ }$ & $\sqrt{ }$ & $\sqrt{ }$ & $\sqrt{ }$ \\
2 & Memopal & $\sqrt{ }$ & $\sqrt{ }$ & $\sqrt{ }$ & $\sqrt{ }$ & $\sqrt{ }$ & $\sqrt{ }$ \\
3 & iBackup & $\sqrt{ }$ & $\sqrt{ }$ & $\sqrt{ }$ & $\sqrt{ }$ & $\sqrt{ }$ & $\sqrt{ }$ \\
4 & Syncplicity & $\sqrt{ }$ & $\sqrt{ }$ & $\sqrt{ }$ & $\sqrt{ }$ & $\sqrt{ }$ & $\sqrt{ }$ \\
5 & FilesAnywhere & $\sqrt{ }$ & $\sqrt{ }$ & $\sqrt{ }$ & $\sqrt{ }$ & $\sqrt{ }$ & $\sqrt{ }$ \\
6 & HostedFTP & $\sqrt{ }$ & $\sqrt{ }$ & $\sqrt{ }$ & $\sqrt{ }$ & $\sqrt{ }$ & $\sqrt{ }$
\end{tabular}

TABLE II: TOP RANKED SOFTWARE COMPLY WITH 'PREFERRED’ CRITERIA

\begin{tabular}{llllllll}
\hline \hline Rank & Service Provider & FP & ACG & MC & UB & UU & Result \\
\hline 1 & DropBox & 2 & 1.98 & $\sqrt{ }$ & $\sqrt{ }$ & $\sqrt{ }$ & 5.2 \\
2 & Memopal & 3 & 2.88 & $\sqrt{ }$ & $\sqrt{ }$ & $\sqrt{ }$ & 1.2 \\
3 & iBackup & $\times$ & 4.97 & $\times$ & $\times$ & $\times$ & -49.7 \\
4 & Syncplicity & 2 & 10.8 & $\sqrt{ }$ & $\sqrt{ }$ & $\sqrt{ }$ & -83 \\
5 & Files Any-where & 1 & 11.9 & $\sqrt{ }$ & $\sqrt{ }$ & $\times$ & -104 \\
6 & HostedFTP & $\times$ & 12 & $\sqrt{ }$ & $\sqrt{ }$ & $\sqrt{ }$ & -105 \\
\hline \hline
\end{tabular}

DropBox (DB), which has the highest rank, is selected as a fundamental element of a system for collaborative teamwork, document management, and on-site information management in SMEs. This is a web and client based software for online 
storage of information. Take into consideration that this application is not a construction management tool but has the potential to be used in construction industry.

DB can be installed in a personal computer (PC), Laptop, and some mobile computing devices such as iPhone, iPad, Android, and also Blackberry phones. When DB is installed in the PC or laptop, a simple folder, namely "My DropBox" will be created in "My Document." By simply copying of any file or folder into "My DropBox" folder, all data will be stored automatically in a secure online server of DB. If the system is not connected to the Internet or the Internet connection is slow, whenever the system becomes online or stable the data will be transferred into the server automatically. Even if the laptop or PC is damaged by fall, electric shock, fire and etc. the worthwhile information of the project will be recovered because project information is kept in the online server. Moreover, one of the features of the DB is capability of information sharing. Therefore, the system can store, backup and share the information among multiple users.

\section{ARCHITECTURE OF THE SySTEM}

The architecture of the system will be discussed in three perspectives as following.

\section{A. Document Management}

Project documents would be organized in 'My DropBox' folder and all documents can be placed in appropriate folders. For instance all documents related to project progress could be organized in a folder which is called "Project Progress." Moreover, by adding metadata such as title, subject, author, category, keywords, comments, and revision number, which is available in the Properties menu of the file in Microsoft Windows, better document management will be achieved.

Because different parties who are involved in a construction project have different information needs, they demand a flexible tool to be customized based on their requirements. DB offers a totally flexible platform by developing structural folders based on organization's demand. Fig. 1 illustrates this document management system for supervisor perspective based on Scott and Assadi [9].

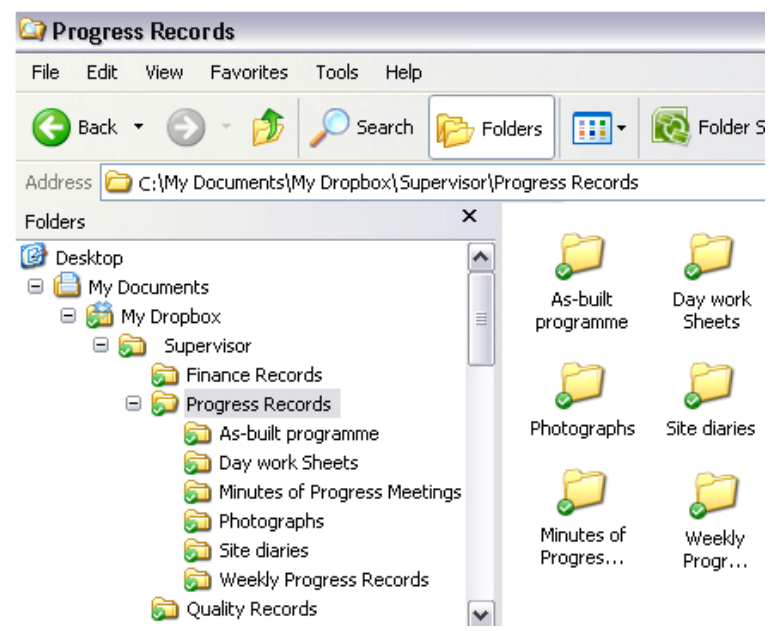

Fig. 1. Example of folder creation for document management adopted from scott and assadi [9].

\section{B. Information Sharing}

The construction industry is a fragmented industry and information sharing is critically necessary for all participants. Information sharing and collaboration can be achieved by sharing the folders. For instance, if a contractor shares the "Progress Report" folder with a client, content of this folder will be transferred into the client's computer. It means that the content of shared folder is the same in both contractor's and client's computer. Once the folder is shared, collaboration will be as easy as copy and paste. The proposed information sharing structure for top management level is demonstrated in Fig. 2.

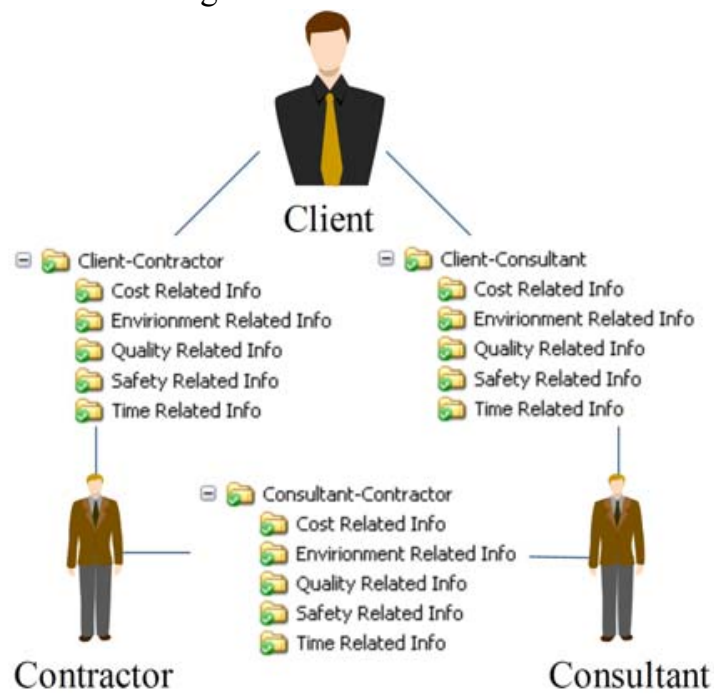

Fig. 2. Proposed document sharing system.

\section{On-site Information Management}

On-site information management is an important issue in construction management. The application of the DB can be installed in the mobile computing tools such as iPhone, iPad, Android and Blackberry phones. The application of DB in these phones is the same as the application of DB in the PCs or laptops. Therefore, foremen, project managers, inspectors, and other parties can benefit from usage of the mobile device to capture, manage, and share on-site information immediately after data entering. Even if there is slow Internet connection in the construction site all information will remain in DB folder and will be synchronized automatically once the connection is stabilized.

\section{Benefits of the System}

1) Simplicity: Users do not need to be trained to use the system. They simply copy and paste the information into DropBox folder in My Document.

2) Functionality: The system can be used as a document management system, groupware system and on-site information management system. All of these functions make it a strong system to be used in construction projects.

3) Flexibility: Document management and information sharing can be customized by construction participants based on their needs and requirements by adding or deleting relevant folders.

4) Affordability: The DB system is free for up to two gigabytes space which seems enough for SMEs, but if 
there is a demand for more space, more space can be purchased.

5) Security: Security of the system is very high because all personnel should sign in using their user name and password. In addition, shared folders can only be viewed by invited users. For instance, if the contractor shares a folder with the client, the consultant does not have access to this folder.

6) Language Independency: There are many languages in the world, but many programs are designed based on English. The DB system is not language dependent and can support any language that the operating system supports. For instance, the client can design a form in Spanish and share it in DB. As long as Spanish is supported by the operating system, other partners can read and edit it simply.

7) Mass Document Input: In construction projects, sometimes it is necessary to share a number of files with other parties. The system is capable of supporting this feature by simply copying and pasting of thousands of files and folders as needed.

8) Document Template: Some of the construction projects have templates for their documents. This system can support any template with any format. Therefore, the user just opens the template in his/her PC or laptop and then after entering data and saving, the file will be updated in his/her PC, the secure server of the DB and also his/her partner's computer.

\section{CONCLUSION}

This paper presented a tool for small- and medium-sized construction companies for collaboration, on-site information management, and document management. DropBox is selected as it complies with the objectives of this study. This software is affordable, simple, functional, and secure. It also supports different document templates, different languages and also mass document input. Unlike ordinary applications, this application can be customised based on companies' information requirements.

Future studies can focus on impact of DropBox in construction companies. Some companies might use the software to be connected to other party. Others, however, might just use it for on-site information management. The practical use of the DropBox is important to be investigated in future studies.

\section{ACKNOWLEDGMENT}

We would like to express our sincere thanks to Universiti Teknologi Malaysia (UTM) for funding the research through the project vote number 77320 .

\section{REFERENCES}

[1] B. Dave, S. Boddy, and L. Koskela, "Improving information flow within the production management system with web services," Proceedings of the 18th Annual Conference of the International Group for Lean Construction. 2010; Haifa, Israel.
[1] Y. Chen and J. Kamara, "The Mechanisms of Information Communication on Construction Sites," FORUM Ejournal, 2008, vol. 8, no. 1, pp. 1-32.

[2] T. Froese, Z. Han, and M. Alldritt, "Study of information technology development for the Canadian construction industry," Canadian Journal of Civil Engineering, 2007, vol. 34, no. 7, pp. 817-829.

[3] J. Irizarry, "Potential application of emerging portable computing platform for information sharing in construction projects," in International Conference on Construction and Real Estate Management (ICCREM), (in CD-ROM). 2008.

[4] J. Irizarry and T. Gill, "Mobile Applications for Information Access on Construction Jobsites," in International Workshop on Computing in Civil Engineering. Austin, TX: ASCE. 2009.

[5] N. Forcada, M. C asala, X. Roca, and M. Gangolells, "Adoption of web databases for document management in SMEs of the construction sector in Spain," Automation in Construction, 2007, 16, no. 4, pp. 411424.

[6] S. L. Chan and N. N. Leung, "Prototype web-based construction project management system," Construction Engineering and Management, 2004, vol. 130, no. 6, pp. 935-943.

[7] P. Chau, "Factors used in the selection of packaged software in small businesses: views of owners and managers," Information \& Management, 1995, vol. 29, no. 2, pp. 71-78.

[8] S. Scott and S. A. Assadi, "Survey of the site records kept by construction supervisors," Construction Management and Economics, 1999, vol. 17, pp. 375-382.

[9] J. M. De la Garza and I. Howitt, "Wireless communication and computing at the construction jobsite," Automation in Construction, 1998, vol. 7, no. 4, pp. 327-347.

[10] K. A. Tenah, "Construction Personnel Role and Information Needs," Construction Engineering and Management, 1986, vol. 112, no. 1, pp. $33-48$.

[11] M. Nourbakhsh, "Mobile application prototype for on-site information management in construction industry," in Civil Engineering. Universiti Teknologi Malaysia: Skudai. 2010.

[12] S. Scott, "Keeping better site records," International Journal of Project Management, 1990, vol. 8, no. 4, pp. 243-249.

[13] B. G. McCullouch and P. Gunn, "Construction Field Data Acquisition with Pen-Based Computers," Construction Engineering and Management, 1993, vol. 119, no. 2, pp. 374-384.

[14] L. C. Wang, Y. C. Lin, and P. H. Lin, "Dynamic mobile RFID-based supply chain control and management system in construction," Advanced Engineering Informatics, 2007, vol. 21, no. 4, pp. 377-390.

[15] L. Y. Liu, "Digital data-collection device for construction site documentation," in Second Congress on Computing in Civil Engineering, ASCE, 1995, pp. 1287-1293.

[16] A. Dong, M. L. Maher, M. J. Kim, N. Gu, and X. Wang, "Construction defect management using a telematic digital workbench," Automation in Construction, 2009, vol. 18, no. 6, pp. 814-824.

[17] R. F. Cox and R. R. A. Issa, "Mobile field data acquisition for construction quality control and ISO 9000 certification," in Proceedings of the Third Congress on Computing in Civil Engineering. San Diego, CA: ASCE. 1996.

[18] K. Kimoto, K. Endo, S. Iwashita, and M. Fujiwara, "The application of PDA as mobile computing system on construction management," Automation in Construction, 2005, vol. 14, no. 4, pp. 500-511.

[19] E. M. Rojas and S A. D. Onger, "FIRS: A vision of the future of building inspection," in Proceedings of the Forth Congress in Computing in Civil Engineering. Philadelphia, PA: ASCE. 1997.

[20] S. Cox, J. Perdomo, and W. Thabet, "Construction field data inspection using pocket PC technology," in International Council for Research and Innovation in Building and Construction CIB w78 2002, Aarhus School of Architecture.

[21] M. Ward, A. Thorpe, A. Price, and C. Wren, "SHERPA: Mobile wireless data capture for piling works," Computer-Aided Civil and Infrastructure Engineering, 2003, vol. 18, no. 4, pp. 299-312.

[22] A. J. Perera and K.. Imriyas, "An integrated construction project cost information system using ms access and MS project," Construction Management and Economics, 2004, vol. 22, no. 2. pp. 203-211.

[23] M. Zhiliang, L. Heng, Q. P. Shen, and Y. Jun, "Using XML to support information exchange in construction projects," Automation in Construction, 2004, vol. 13, no. 5, pp. 629-637.

[24] S. O. Cheung, H. C. H. Suen, and Ch K. K. W. Eung, "PPMS: A web-based construction project performance monitoring system," Automation in Construction, 2004, vol. 13, no. 3, pp. 361-376. 